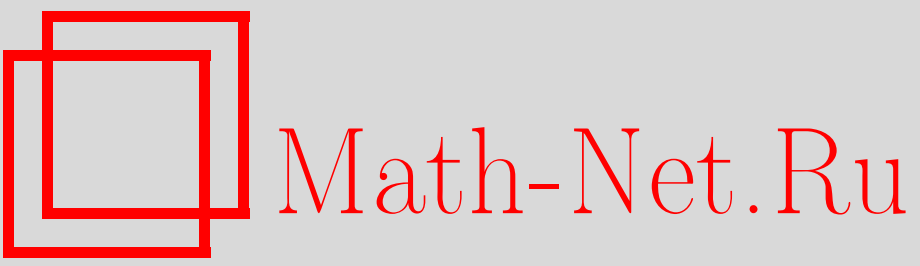

И. И. Бодренко, Об условиях параллельности тензора нормальной кривизны подмногообразий, Итоги науки и техн. Сер. Соврем. мат. и ее прил. Темат. обз., 2020, том 181, 3-8

DOI: https://doi.org/10.36535/0233-6723-2020-181-3-8

Использование Общероссийского математического портала Math-Net.Ru подразумевает, что вы прочитали и согласны с пользовательским соглашением

http://www . mathnet.ru/rus/agreement

Параметры загрузки:

IP : 34.239 .49 .27

26 апреля 2023 г., 13:43:17 


\title{
ОБ УСЛОВИЯХ ПАРАЛЛЕЛЬНОСТИ ТЕНЗОРА НОРМАЛЬНОЙ КРИВИЗНЫ ПОДМНОГООБРАЗИЙ
}

\author{
(c) 2020 г. $\quad$ И. И. БОДРЕНКО
}

\begin{abstract}
АннотАция. В работе получены достаточные условия параллельности тензора нормальной кривизны подмногообразий в пространствах постоянной кривизны.

Ключевые слова: подмногообразие, пространство постоянной кривизны, вторая фундаментальная форма, тензор нормальной кривизны, вектор нормальной кривизны, вектор нормального кручения.
\end{abstract}

\section{CONDITIONS FOR THE PARALLELISM}

\section{OF THE NORMAL CURVATURE TENSOR OF SUBMANIFOLDS}

\section{(c) 2020 I. I. BODRENKO}

\begin{abstract}
In this paper, sufficient conditions for the parallelism of the normal curvature tensor of submanifolds in spaces of constant curvature are obtained.

Keywords and phrases: submanifold, space of constant curvature, second fundamental form, normal curvature tensor, normal curvature vector, normal torsion vector.
\end{abstract}

AMS Subject Classification: 53B25, 53C40

1. Введение. Пусть $M^{n+p}-(n+p)$-мерное $(n \geqslant 2, p \geqslant 2)$ гладкое риманово многообразие, $\tilde{g}$ - риманова метрика на $M^{n+p}, \tilde{\nabla}$ - риманова связность, согласованная с $\tilde{g}, \tilde{R}$ - преобразование кривизны связности $\tilde{\nabla}$. Пусть $F^{n}-n$-мерное гладкое подмногообразие в $M^{n+p}$. Обозначим через $g$ индуцированную риманову метрику на $F^{n}$, через $\nabla$ - риманову связность на $F^{n}$, согласованную с $g$, через $R$-преобразование кривизны связности $\nabla$. Вторая фундаментальная форма $b$ подмногообразия $F^{n} \subset M^{n+p}$ определяется по формуле Гаусса

$$
\tilde{\nabla}_{X} Y=\nabla_{X} Y+b(X, Y)
$$

для любых векторных полей $X, Y$, касательных к $F^{n}$.

Пусть $D$ - нормальная связность, $R^{\perp}$ - тензор нормальной кривизны подмногообразия $F^{n} \subset$ $M^{n+p}$. Нормальная связность $D$ называется плоской, если $R^{\perp} \equiv 0$ на $F^{n}$.

Для любого векторного поля $\xi$, нормального к $F^{n}$, и любого векторного поля $X$, касательного к $F^{n}$, формула Вейнгартена имеет вид

$$
\tilde{\nabla}_{X} \xi=-A_{\xi} X+D_{X} \xi
$$

где $A_{\xi}$ - второй фундаментальный тензор, соответствующий $\xi$.

Оператор Вейнгартена $A_{\xi}$ удовлетворяет равенству

$$
\tilde{g}(b(X, Y), \xi)=g\left(A_{\xi} X, Y\right)
$$

для любых векторных полей $X, Y$, касательных к $F^{n}$, и векторного поля $\xi$, нормального к $F^{n}$. 
Обозначим через $\bar{\nabla}=\nabla \oplus D$ связность ван дер Вардена-Бортолотти. Ковариантные производные $\bar{\nabla} b,(\bar{\nabla} A)_{\xi}, \bar{\nabla} R^{\perp}$ определяются соответственно равенствами

$$
\begin{gathered}
\left(\bar{\nabla}_{X} b\right)(Y, Z)=D_{X}(b(Y, Z))-b\left(\nabla_{X} Y, Z\right)-b\left(Y, \nabla_{X} Z\right), \\
\left(\bar{\nabla}_{X} A\right)_{\xi} Y=\nabla_{X}\left(A_{\xi} Y\right)-A_{\xi}\left(\nabla_{X} Y\right)-A_{D_{X} \xi} Y \\
\left(\bar{\nabla}_{X} R^{\perp}\right)(Y, Z) \xi=D_{X}\left(R^{\perp}(Y, Z) \xi\right)-R^{\perp}\left(\nabla_{X} Y, Z\right) \xi-R^{\perp}\left(Y, \nabla_{X} Z\right) \xi-R^{\perp}(Y, Z) D_{X} \xi,
\end{gathered}
$$

для любых векторных полей $X, Y, Z$, касательных к $F^{n}$, и векторного поля $\xi$, нормального к $F^{n}$.

Уравнения Гаусса, Петерсона-Кодацци и Риччи имеют соответственно вид

$$
\begin{gathered}
\tilde{R}(X, Y, Z, W)=R(X, Y, Z, W)+\tilde{g}(b(X, Z), b(Y, W))-\tilde{g}(b(X, W), b(Y, Z)), \\
(\tilde{R}(X, Y) Z)^{\perp}=\left(\bar{\nabla}_{X} b\right)(Y, Z)-\left(\bar{\nabla}_{Y} b\right)(X, Z), \\
\tilde{R}(X, Y, \xi, \eta)=R^{\perp}(X, Y, \xi, \eta)-g\left(\left[A_{\xi}, A_{\eta}\right] X, Y\right)
\end{gathered}
$$

для любых векторных полей $X, Y, Z, W$, касательных к $F^{n}$, и векторных полей $\xi, \eta$, нормальных к $F^{n}$.

Риманово многообразие $M^{n+p}$ называется пространством постоянной кривизны $\tilde{c}(\tilde{c}=$ const $)$ и обозначается $M^{n+p}(\tilde{c})$, если преобразование кривизны $\tilde{R}$ удовлетворяет условию

$$
\tilde{R}(\tilde{X}, \tilde{Y}) \tilde{Z}=\tilde{c}(\tilde{g}(\tilde{Y}, \tilde{Z}) \tilde{X}-\tilde{g}(\tilde{X}, \tilde{Z}) \tilde{Y})
$$

для любых векторных полей $\tilde{X}, \tilde{Y}, \tilde{Z}$, касательных к $M^{n+p}$.

В силу (3) для подмногообразия $F^{n}$ в пространстве постоянной кривизны $M^{n+p}(\tilde{c})$ уравнения Гаусса, Петерсона-Кодацци и Риччи имеют соответственно вид

$$
\begin{gathered}
R(X, Y, Z, W)=\tilde{c}(g(X, W) g(Y, Z)-g(X, Z) g(Y, W))+ \\
+\tilde{g}(b(X, W), b(Y, Z))-\tilde{g}(b(X, Z), b(Y, W)), \\
\left(\bar{\nabla}_{X} b\right)(Y, Z)=\left(\bar{\nabla}_{Y} b\right)(X, Z) \\
R^{\perp}(X, Y, \xi, \eta)=g\left(\left[A_{\xi}, A_{\eta}\right] X, Y\right)
\end{gathered}
$$

для любых векторных полей $X, Y, Z, W$, касательных к $F^{n}$, и векторных полей $\xi, \eta$, нормальных к $F^{n}$.

Определение 1. Тензор нормальной кривизны $R^{\perp} \neq 0$ называется параллельным, если для любых векторных полей $X, Y, Z$, касательных к $F^{n}$, и векторного поля $\xi$, нормального к $F^{n}$, выполняется равенство

$$
\left(\bar{\nabla}_{Z} R^{\perp}\right)(X, Y) \xi=0 .
$$

Замечание 1. Основным инвариантом нормальной связности $D$ двумерной поверхности $F^{2}$ в евклидовом пространстве $E^{4}$ является гауссово кручение $\varkappa$. В [11] установлено, что поверхность $F^{2} \subset E^{4}$ имеет постоянное гауссово кручение $\varkappa \equiv$ const $\neq 0$ тогда и только тогда, когда тензор нормальной кривизны $R^{\perp} \neq 0$ параллелен.

В геометрии погруженных многообразий значительное место занимают исследования подмногообразий со специальными свойствами второй фундаментальной формы. Вторая фундаментальная форма $b \neq 0$ называется параллельной, если $\bar{\nabla} b=0$.

Определение 2. Вторая фундаментальная форма $b \neq 0$ называется рекуррентной, если на $F^{n}$ существует такая 1-форма $\mu$, что $\bar{\nabla} b=\mu \otimes b$.

Свойства кэлеровых подмногообразий с рекуррентной второй фундаментальной формой в пространствах постоянной голоморфной секционной кривизны исследовались в [1].

Определение 3. Вторая фундаментальная форма $b \neq 0$ называется циклически рекуррентной, если на $F^{n}$ существует такая 1-форма $\mu$, что

$$
\left(\bar{\nabla}_{X} b\right)(Y, Z)=\mu(X) b(Y, Z)+\mu(Y) b(Z, X)+\mu(Z) b(X, Y)
$$

для любых векторных полей $X, Y, Z$, касательных к $F^{n}$. 
Подмногообразия $F^{n} \subset M^{n+p}(\tilde{c})$ с циклически рекуррентной второй фундаментальной формой $b \neq 0$ (или короче циклически рекуррентные подмногообразия) являются естественными обобщениями поверхностей Дарбу в трехмерном евклидовом пространстве $E^{3}$ (см. [8]). Класс циклически рекуррентных подмногообразий содержит подклассы параллельных подмногообразий и непараллельных рекуррентных подмногообразий, но не исчерпывается ими. Некоторые свойства гиперповерхностей $F^{n}$ с циклически рекуррентной непараллельной второй фундаментальной формой в евклидовых пространствах $E^{n+1}$ установлены в $[2,6,7]$. Подмногообразия $F^{n}$ с непараллельной рекуррентной второй фундаментальной формой $b \neq 0$ и плоской нормальной связностью в пространствах постоянной кривизны $M^{n+p}(\tilde{c})$ описаны в [9]. Циклически рекуррентные подмногообразия $F^{n}$ с плоской нормальной связностью в евклидовых пространствах $E^{n+p}$ классифицированы в $[5,10]$.

Пусть $x \in F^{n}$ - произвольная точка, $T_{x} F^{n}$ и $N_{x} F^{n}$ - соответственно касательное и нормальное пространства к $F^{n}$ в $x$ :

$$
T_{x} M^{n+p}(\tilde{c})=T_{x} F^{n} \oplus N_{x} F^{n} .
$$

Пусть $\gamma$ - кривая на $F^{n}$, проходящая через точку $x$ в направлении вектора $t \in T_{x} F^{n}, T_{(\gamma)}$ - единичное векторное поле, касательное к $\gamma$. Введем обозначение $T_{(\gamma)}(x)=\tau$, где $\tau=t /|t|$. Обозначим через $\tilde{X}_{(\gamma)}$ поле вектора первой кривизны кривой $\gamma$ в $M^{n+p}(\tilde{c})$ :

$$
\tilde{\nabla}_{T_{(\gamma)}} T_{(\gamma)}=\tilde{X}_{(\gamma)}
$$

Пусть $k_{T} \in T_{x} F^{n}$ и $k_{N} \in N_{x} F^{n}$ - соответственно касательная и нормальная составляющие вектора $\tilde{X}_{(\gamma)}(x) \in T_{x} M^{n+p}(\tilde{c})$. Тогда в силу формулы Гаусса (1) имеем

$$
k_{T}=\nabla_{T_{(\gamma)}} T_{(\gamma)}(x), \quad k_{N}=b(\tau, \tau) .
$$

Вектор $k_{N} \in N_{x} F^{n}$, нормальный к $F^{n}$ в точке $x$, зависит от точки $x$ и направления $t$ и не зависит от выбора кривой $\gamma$. Будем использовать обозначение $k_{N} \equiv k_{N}(x, t)$.

Определение 4. Вектор $k_{N}(x, t) \in N_{x} F^{n}$ называется вектором нормальной кривизны подмногообразия $F^{n} \subset M^{n+p}(\tilde{c})$ в точке $x \in F^{n}$ по направлению $t \in T_{x} F^{n}$.

Обозначим через $\gamma(x, t)$ геодезическую на $F^{n}$, проходящую через точку $x \in F^{n}$ в направлении $t \in T_{x} F^{n}$. Пусть в каждой точке $y \in \gamma(x, t)$ в $M^{n+p}(\tilde{c})$ однозначно определен единичный вектор первой нормали $\eta=\eta(y)$. Обозначим через $\left|k_{g}(x, t)\right|$ первую кривизну геодезической $\gamma(x, t)$ в $M^{n+p}(\tilde{c})$, вычисленную в точке $x$. Тогда

$$
\tilde{\nabla}_{T_{(\gamma)}} T_{(\gamma)}=\left|k_{g}\left(y, T_{(\gamma)}(y)\right)\right| \eta(y) \quad \forall y \in \gamma(x, t),
$$

где $T_{(\gamma)}=T_{(\gamma)}(y)$ - единичный касательный вектор к геодезической $\gamma(x, t)$ в точке $y, T_{(\gamma)}(x)=$ $t /|t|,\left|k_{g}\left(y, T_{(\gamma)}(y)\right)\right|$ - первая кривизна геодезической $\gamma(x, t)$ в точке $y$. Учитывая, что $\nabla_{T_{(\gamma)}} T_{(\gamma)}=$ 0 вдоль геодезической $\gamma(x, t)$, имеем

$$
\left|k_{g}\left(y, T_{(\gamma)}(y)\right)\right| \eta(y)=b\left(T_{(\gamma)}, T_{(\gamma)}\right) \quad \forall y \in \gamma(x, t) .
$$

Так как $\eta(y) \in N_{y} F^{n}$, то, используя формулу Вейнгартена $(2)$, вдоль $\gamma(x, t)$ получим

$$
\tilde{\nabla}_{T_{(\gamma)}} \eta=-A_{\eta} T_{(\gamma)}+D_{T_{(\gamma)}} \eta
$$

Обозначим через $\varkappa_{N} \in N_{x} F^{n}$ нормальную составляющую вектора $\tilde{\nabla}_{T_{(\gamma)}} \eta$ в точке $x$, т.е. $\varkappa_{N}=$ $D_{T_{(\gamma)}} \eta(x)$. Вектор $\varkappa_{N} \in N_{x} F^{n}$, нормальный к $F^{n}$ в точке $x$, зависит от точки $x \in F^{n}$ и направления $t \in T_{x} F^{n}$. Будем использовать обозначение $\varkappa_{N} \equiv \varkappa_{N}(x, t)$.

Определение 5. Вектор $\varkappa_{N}(x, t) \in N_{x} F^{n}$ называется вектором нормального кручения подмногообразия $F^{n} \subset M^{n+p}(\tilde{c})$ в точке $x \in F^{n}$ по направлению $t \in T_{x} F^{n}$.

Определение 6. Инварианты $\left|k_{N}(x, t)\right|$ и $\left|\varkappa_{N}(x, t)\right|$ называются соответственно нормальной кривизной и нормальным кручением подмногообразия $F^{n} \subset M^{n+p}(\tilde{c})$ в точке $x \in F^{n}$ по направлению $t \in T_{x} F^{n}$. 
Свойства двумерных поверхностей $F^{2}$ с нулевым нормальным кручением в четырехмерном евклидовом пространстве $E^{4}$ изучались в [13]. Исследования свойств подмногообразий $F^{n}$ с нулевым нормальным кручением в евклидовых пространствах $E^{n+p}$ для произвольных $n$ и $p$ продолжены в [4].

Замечание 2. Для двумерных поверхностей в четырехмерном римановом пространстве понятие нормального кручения поверхности $F^{2}$ в точке $x$ по неасимптотическому направлению $t$ ввел В. Т. Фоменко в [12]. Понятие вектора нормального кручения подмногообразия $F^{n} \subset M^{n+p}(\tilde{c})$ в точке $x \in F^{n}$ по неасимптотическому направлению $t \in T_{x} F^{n}$ в случае $n \geqslant 2, p \geqslant 2$ введено в [3].

Имеет место следующая формула (см. [3]):

$$
\varkappa_{N}(x, t)=\frac{\left(\bar{\nabla}_{\tau} b\right)(\tau, \tau)}{|b(\tau, \tau)|}-\frac{b(\tau, \tau) \tilde{g}\left(b(\tau, \tau),\left(\bar{\nabla}_{\tau} b\right)(\tau, \tau)\right)}{|b(\tau, \tau)|^{3}},
$$

где $\tau=t /|t|$; формула (7) имеет смысл, если $t$ - неасимптотическое направление.

На $F^{n} \subset M^{n+p}(c)$ определены специальные классы нормальных векторных полей в терминах второй фундаментальной формы $b$.

Определение 7. Нормальное векторное поле $b(T, T)$, определенное для любого единичного векторного поля $T$, касательного к $F^{n}$, называется полем вектора нормальной кривизны подмногообразия $F^{n} \subset M^{n+p}(\tilde{c})$ в направлении $T$.

Пусть $f$ - функция на $F^{n}, f(x) \neq 0$ для всех $x \in F^{n}$. Рассмотрим вдоль кривой $\gamma$ нормальное векторное поле

$$
f_{\mid \gamma} b\left(T_{(\gamma)}, T_{(\gamma)}\right)
$$

где $f_{\mid \gamma}(u)=f(u)$ для всех $u \in \gamma$.

Определение 8. Поле вектора нормальной кривизны $F^{n} \subset M^{n+p}(\tilde{c})$ вдоль кривой $\gamma$ называется рекуррентным, если существует такая функция $f$ на $F^{n}\left(f(x) \neq 0\right.$ для всех $\left.x \in F^{n}\right)$, что

$$
D_{T_{(\gamma)}}\left(f_{\mid \gamma} b\left(T_{(\gamma)}, T_{(\gamma)}\right)\right)=0 .
$$

Замечание 3. Для подмногообразий $F^{n}$ в евклидовом пространстве $E^{n+p}$ понятие рекуррентного вектора нормальной кривизны ввел В. Т. Фоменко в [14]. Понятие рекуррентного поля вектора нормальной кривизны подмногообразия $F^{n}$ вдоль кривой $\gamma$ в пространстве постоянной кривизны $M^{n+p}(\tilde{c})$ введено в [3].

2. Формулировка результатов. Справедливы следующие утверждения.

Теорема 1. Пусть нормальная связность поверхности $F^{2}$ в пространстве $M^{4}(\tilde{c})$ постоянной кривизны не является плоской $\left(R^{\perp} \neq 0\right)$ и поверхность $F^{2}$ не имеет асимптотических направлений. Если поверхность $F^{2} \subset M^{4}(\tilde{c})$ имеет ииклически рекуррентную вторую фундаментальную форму $b$, то тензор нормальной кривизны $R^{\perp}$ поверхности $F^{2}$ параллелен.

Теорема 2. Пусть нормальная связность поверхности $F^{2}$ в пространстве $M^{4}(\tilde{c})$ постоянной кривизны не является плоской $\left(R^{\perp} \neq 0\right)$ и поверхность $F^{2}$ не имеет асимптотических направлений. Если поверхность $F^{2} \subset M^{4}(\tilde{c})$ имеет рекуррентное поле вектора нормальной кривизны вдоль каждой геодезической $\gamma(x, t)$, то тензор нормальной кривизны $R^{\perp}$ поверхности $F^{2}$ параллелен.

Теорема 3. Пусть нормальная связность подмногообразия $F^{n}, n \geqslant 2$, в пространстве $M^{n+p}(\tilde{c})$ постоянной кривизны не является плоской $\left(R^{\perp} \neq 0\right)$ и подмногообразие $F^{n}$ не имеет асимптотических направлений. Если $F^{n} \subset M^{n+p}(\tilde{c})$ имеет рекуррентную вторую фундаментальную форму $b$, то тензор нормальной кривизны $R^{\perp}$ подмногообразия $F^{n}$ параллелен. 


\section{3. Основные леммы.}

Лемма 1. Пусть $F^{n}$ - подмногообразие в пространстве постоянной кривизны $M^{n+p}(\tilde{c})$. Тогда для любых векторных полей $X, Y, Z$, касательных к $F^{n}$, и векторного поля $\xi$, нормального к $F^{n}$, справедливо равенство

$$
\left(\bar{\nabla}_{Z} R^{\perp}\right)(X, Y) \xi=\left(\bar{\nabla}_{Z} b\right)\left(X, A_{\xi} Y\right)+b\left(X,\left(\bar{\nabla}_{Z} A\right)_{\xi} Y\right)-\left(\bar{\nabla}_{Z} b\right)\left(Y, A_{\xi} X\right)-b\left(Y,\left(\bar{\nabla}_{Z} A\right)_{\xi} X\right) .
$$

Используя лемму 1 , получим следующее утверждение.

Лемма 2. Пусть $F^{n}$ - подмногообразие в пространстве постоянной кривизны $M^{n+p}(\tilde{c})$. Тогда для любых векторных полей $X, Y, Z$, касательных к $F^{n}$, и векторных полей $\xi, \eta$, нормальных к $F^{n}$, справедливо равенство

$$
\left(\bar{\nabla}_{Z} R^{\perp}\right)(X, Y, \xi, \eta)=g\left(\left[\left(\bar{\nabla}_{Z} A\right)_{\xi}, A_{\eta}\right] X, Y\right)+g\left(\left[A_{\xi},\left(\bar{\nabla}_{Z} A\right)_{\eta}\right] X, Y\right)
$$

Лемма 3. Пусть нормальная связность поверхности $F^{2}$ в пространстве $M^{4}(\tilde{c})$ постоянной кривизны не является плоской $\left(R^{\perp} \neq 0\right)$ и поверхность $F^{2}$ не имеет асимптотических направлений. Если на $F^{2}$ выполняется соотношение

$$
\left|\varkappa_{N}(x, t)\right| \equiv 0 \quad \forall x \in F^{2}, \forall t \in T_{x} F^{2},
$$

то на $F^{2}$ справедливо равенство

$$
\left(\bar{\nabla}_{X} A\right)_{\xi}=0
$$

для любого векторного поля $X$, касательного к $F^{2}$, и векторного поля $\xi$, нормального к $F^{2}$.

Доказательство. В силу [12, теорема 2] соотношение (8) выполняется на $F^{2}$ тогда и только тогда, когда выполняются следующие условия:

(i) $F^{2}$ лежит в пространстве $M^{4}(\tilde{c})$ постоянной положительной кривизны $\tilde{c}=3 \lambda_{0}^{2}, \lambda_{0}=$ const,

(ii) $F^{2}$ имеет постоянную положительную внутреннюю кривизну $K_{i}=\lambda_{0}^{2}$,

(iii) индикатриса нормальной кривизны поверхности $F^{2}$ в каждой точке $x \in F^{2}$ является окружностью радиуса $\lambda_{0}>0$ с центром в точке $x$.

Отсюда, учитывая уравнение Гаусса (4), на $F^{2}$ имеем

$$
\left(\bar{\nabla}_{Z} b\right)(X, Y)=0
$$

для любых векторных полей $X, Y, Z$, касательных к $F^{2}$. Учитывая, что (10) равносильно равенству (9), приходим к утверждению леммы.

Доказательство теоремь 1. Если поверхность $F^{2}$ без асимптотических направлений в пространстве постоянной кривизны $M^{4}(\tilde{c})$ имеет циклически рекуррентную вторую фундаментальную форму $b$, то в соответствии с [3, теорема 1] поверхность $F^{2}$ имеет нулевой вектор нормального кручения $\varkappa_{N}(x, t) \equiv 0$ в каждой точке $x \in F^{2}$ по любому направлению $t \in T_{x} F^{2}$. Тогда на $F^{2}$ выполняется условие (8). Следовательно, в силу леммы 3 на $F^{2}$ справедливо равенство (9). Применение леммы 2 завершает доказательство теоремы.

Доказательство теоремы 2. Если поверхность $F^{2}$ без асимптотических направлений в $M^{4}(\tilde{c})$ имеет рекуррентное поле вектора нормальной кривизны вдоль каждой геодезической $\gamma(x, t)$, то в силу [3, теорема 2] поверхность $F^{2}$ имеет нулевой вектор нормального кручения $\varkappa_{N}(x, t) \equiv 0$ в каждой точке $x \in F^{2}$ по любому направлению $t \in T_{x} F^{2}$. Тогда на $F^{2}$ выполняется условие (8). Отсюда, в силу леммы 3 , из леммы 2 получим утверждение теоремы.

Доказательство теоремы 3. Пусть $F^{n} \subset M^{n+p}(\tilde{c})$ имеет рекуррентную вторую фундаментальную форму $b$, т.е. на $F^{n}$ существует такая 1-форма $\mu$, что выполняется соотношение

$$
\left(\bar{\nabla}_{Z} b\right)(X, Y)=\mu(Z) b(X, Y)
$$

для любых векторных полей $X, Y, Z$, касательных к $F^{n}$. Условие (11) эквивалентно условию

$$
\left(\bar{\nabla}_{X} A\right)_{\xi}=\mu(X) A_{\xi}
$$

для любого векторного поля $X$, касательного к $F^{n}$, и векторного поля $\xi$, нормального к $F^{n}$. 
В силу уравнений Риччи (6) из условия $R^{\perp} \neq 0$ следует, что в каждой точке $x \in F^{n}$ найдутся такие векторы $t_{1}, t_{2}, t_{3}, t_{4} \in T_{x} F^{n}$, что векторы $b\left(t_{1}, t_{2}\right), b\left(t_{3}, t_{4}\right) \in N_{x} F^{n}$ линейно независимы. Рассмотрим линейную комбинацию векторов $\mu\left(t_{1}\right) \mu\left(t_{2}\right) b\left(t_{3}, t_{4}\right)-\mu\left(t_{3}\right) \mu\left(t_{4}\right) b\left(t_{1}, t_{2}\right) \in N_{x} F^{n}$. Учитывая уравнения Петерсона-Кодацци (5) и соотношение (11), получим:

$$
\mu\left(t_{1}\right) \mu\left(t_{2}\right) b\left(t_{3}, t_{4}\right)-\mu\left(t_{3}\right) \mu\left(t_{4}\right) b\left(t_{1}, t_{2}\right)=\mu\left(t_{1}\right) \mu\left(t_{3}\right) b\left(t_{2}, t_{4}\right)-\mu\left(t_{3}\right) \mu\left(t_{4}\right) b\left(t_{1}, t_{2}\right)=0 .
$$

Отсюда в силу линейной независимости векторов $b\left(t_{1}, t_{2}\right), b\left(t_{3}, t_{4}\right) \in N_{x} F^{n}$ имеем

$$
\mu\left(t_{1}\right) \mu\left(t_{2}\right)=\mu\left(t_{3}\right) \mu\left(t_{4}\right)=0 .
$$

Тогда для любого вектора $t \in T_{x} F^{n}$ выполняются равенства

$$
\mu(t) b\left(t_{1}, t_{2}\right)=\mu\left(t_{1}\right) b\left(t, t_{2}\right)=\mu\left(t_{2}\right) b\left(t_{1}, t\right)=0 .
$$

Следовательно, $\mu(t)=0$ для всех $t \in T_{x} F^{n}$, и из (12) получим равенство

$$
\left(\bar{\nabla}_{X} A\right)_{\xi}=0
$$

для любого векторного поля $X$, касательного к $F^{n}$, и векторного поля $\xi$, нормального к $F^{n}$. Применение леммы 2 завершает доказательство теоремы.

\section{СПИСОК ЛИТЕРАТУРЫ}

1. Бодренко И. И. Некоторые свойства кэлеровых подмногообразий с рекуррентными тензорными полями// Вестн. Волгоград. ун-та. Сер. 1. Мат. Физ. - 2006. - 10, № 1. - С. 11-21.

2. Бодренко И. И. О гиперповерхностях с циклически рекуррентной второй фундаментальной формой в евклидовом пространстве// Вестн. Волгоград. ун-та. Сер. 1. Мат. Физ. - 2010. - 13. - С. 23-35.

3. Бодренко И. И. О подмногообразиях с нулевым вектором нормального кручения в пространствах постоянной кривизны// в кн.: Ломоносовские чтения на Алтае. - Барнаул, 2015. - С. 461-465.

4. Бодренко И. И. О подмногообразиях с нулевым нормальным кручением в евклидовом пространстве// Сиб. мат. ж. - 1994. - 35, № 3. - С. 527-536.

5. Бодренко И. И. О подмногообразиях с циклически рекуррентной второй фундаментальной формой в евклидовых пространствах// Обозр. прикл. пром. мат. - 2011. - 18, № 5. - С. 746.

6. Бодренко И. И. Об аналоге поверхностей Дарбу в многомерных евклидовых пространствах// Вестн. Волгоград. ун-та. Сер. 1. Мат. Физ. - 2013. - № 1 (18). - С. 24-30.

7. Бодренко И. И. Обобщение теоремы Бонне о поверхностях Дарбу// Мат. заметки. - 2014. - 95, № 6. - C. $812-820$.

8. Бодренко И. И. Обобщенные поверхности Дарбу в пространствах постоянной кривизны. - Saarbrücken: LAP LAMBERT, 2013.

9. Бодренко И. И. Подмногообразия с рекуррентной второй фундаментальной формой в пространствах постоянной кривизны// Обозр. прикл. пром. мат. - 2007. - 14, № 4. - С. 679-682.

10. Бодренко И. И. Строение подмногообразий с циклически рекуррентной второй фундаментальной формой в евклидовом пространстве// Вестн. Волгоград. ун-та. Сер. 1. Мат. Физ. - 2011. - № 1 (14). C. $10-17$.

11. Бодренко И. И. Характеристический признак поверхностей с постоянным гауссовым кручением в $E^{4} / /$ Вестн. Волгоград. ун-та. Сер. 1. Мат. Физ. - 2013. - № 2 (19). - С. 13-17.

12. Фоменко В. Т. Классификация двумерных поверхностей с нулевым нормальным кручением в четырехмерном пространстве постоянной кривизны// Мат. заметки. -2004 . -75 , № 5. - С. 744-756.

13. Фоменко В. Т. Некоторые свойства двумерных поверхностей с нулевым нормальным кручением в $E^{4} / /$ Мат. сб. - 1978. - 106 (148), № 4 (8). - С. 589-603.

14. Фоменко В. Т. Об одном обобщении поверхностей Дарбу// Мат. заметки. $-1990 .-48$, № 2. - С. 107113.

Бодренко Ирина Ивановна

ООО «Интерактивные системы», Волгоград

E-mail: irina@bodrenko.org 